\title{
Gestión empresarial, nivel de competitividad y productividad de empresas del sector textil de Huancayo
}

\author{
Business management, competitiveness level and productivity of \\ textile companies from Huancayo
}

Gustavo Loayza Acosta', Sonia Curasma Quispe².

Universidad Continental

\section{RESUMEN}

Objetivos: Determinar el nivel de competitividad de empresas del sector textil con potencial exportador de la provincia de Huancayo, Perú; promover el desarrollo de las áreas más críticas de gestión de las empresas objeto de estudio de acuerdo con el resultado del mapa de competitividad. Métodos: La investigación fue aplicada, con diseño descriptivo. La recolección de datos fue a través de la aplicación del mapa de competitividad empresarial, que permitió obtener información del nivel de competitividad y productividad de la empresa. La muestra estuvo constituida por 15 empresas participantes en el Programa de Training en Gestión Exportadora (PTGE) de Promperú. Las mediciones se realizaron al inicio y al final, para luego determinar los respectivos diagnósticos de competitividad de la empresa. Resultados: Las empresas incrementaron el nivel de competitividad de 2,96 a 3,67 puntos sobre un total de 5 puntos. El incremento porcentual por áreas fue de $60 \%$ a $73 \%$ en planeamiento estratégico, de $62 \%$ a $74 \%$ en producción y operaciones, de $64 \%$ a $76 \%$ en aseguramiento de la calidad, de $56 \%$ a $74 \%$ en comercialización, de $66 \%$ a $74 \%$ en contabilidad y finanzas, de $66 \%$ a $75 \%$ en recursos humanos, de $47 \%$ a $75 \%$ en gestión ambiental, y de $42 \%$ a $63 \%$ en sistemas de información. Conclusiones: Se incrementó la competitividad de las empresas, $18 \%$ en comercialización, $21 \%$ en sistemas de información y $28 \%$ en gestión ambiental, por citar las áreas donde se alcanzó un mejor desempeño.

Palabras Clave: Gestión empresarial, competitividad, productividad, gestión exportadora, comercialización, gestión ambiental, sistemas de Información.

1 Economista, director de la Escuela Académica de Economía y docente investigador de la Universidad Continental.

2 Bach, en Economía, consultora de Promperú en la región Junín, Perú. 


\section{ABSTRACT}

Objectives: To determine the competitiveness level of textile companies with export potential in Huancayo province, Peru; to promote the development of the most critical management areas of companies which are study subject according to the results of the competitiveness map. Methods: The research was applied with pre-experimental design. Data collection was through the business competitiveness map application, which allowed to obtain information about the competitiveness and productivity level of the company. The sample consisted of 15 companies that participate in the Training Program in Export Management (PTGE) from Promperú. The measures were taken at the beginning and at the end, then to determine the respective competitiveness diagnoses of the company. Results: Companies increased the competitiveness level from 2,96 to 3,67 points out of a total of 5 points. The percentage increase by areas was from $60 \%$ to $73 \%$ on strategic planning, $62 \%$ to $74 \%$ on production and operations, $64 \%$ to $76 \%$ on quality assurance, $56 \%$ to $74 \%$ on marketing, $66 \%$ to $74 \%$ on accounting and finance, $66 \%$ to $75 \%$ in human resources, $47 \%$ to $75 \%$ in environmental management, and $42 \%$ to $63 \%$ in information systems. Conclusions: Competitiveness of PTGE's companies steadily increased: $18 \%$ in marketing, $21 \%$ in information systems and $28 \%$ in environmental management. To cite areas where a better performance was achieved. Specialty labor skills and soft skills in students who participated in PTGE increased.

Keywords: Business management, competitiveness, productivity, export management, marketing, environmental management, information systems.

\section{INTRODUCCIÓN}

El Programa de Training en Gestión Exportadora (PTGE) surge como necesidad de dar continuidad en la mejora del nivel competitivo (1) de empresas del sector textil con potencial exportador, que no cuentan con colaboradores que puedan impulsar la viabilidad a un plan de mejora de la competitividad de estas, principalmente por el alto costo que ellos representan (remuneraciones). El Estado a través de la Comisión de Promoción del Perú para la Exportación y Turismo (Promperú) solo podía financiar asistencia técnica pero no la permanencia de colaboradores que le ayuden a la implementación de planes de mejora. En este contexto, se planteó a las empresas agrupadas en el Consejo Ejecutivo Regional de Exportaciones Junín (CERX Junín) un triángulo macroeconómico (Figura 1) con tres instituciones: Promperú, por el Estado; CERX Junín, por el sector empresarial; y la Universidad Continental y el Instituto Continental representando a la Organización Educativa Continental (OEC), por la academia con la aprobación de los directivos de estas instituciones se inició el PTGE.

A través de la interacción de las empresas, instituciones educativas y el Estado se buscó que las empresas exportadoras logren una gestión empresarial con resultados y objetivos en exportación continua que impacten en el desempeño competitivo de las empresas del PTGE en mercados internacionales.

Un análisis de dimensión nacional realizado por la Universidad del Pacífico (2), indica que el sector textil peruano tiene un enorme potencial por la "tradición ancestral de más de 3000 años", que puede concretarse si se logra formar una cadena de valor sostenible siempre que esté soportada por un eficiente proceso productivo.

En la región Junín existe un diagnóstico y prospectiva competitiva (3) sobre sus actividades económicas potenciales, pero de manera inexplicable entre estas no se ha incluido el sector textil, a pesar de contar con una cantidad significativa de empresas dedicadas al rubro.

En el 2005, el Ministerio de Comercio Exterior elaboró el Plan Estratégico Regional Exportador (PERX) (4) en el cual se desarrolló el Plan Operativo Sectorial (POS) de 


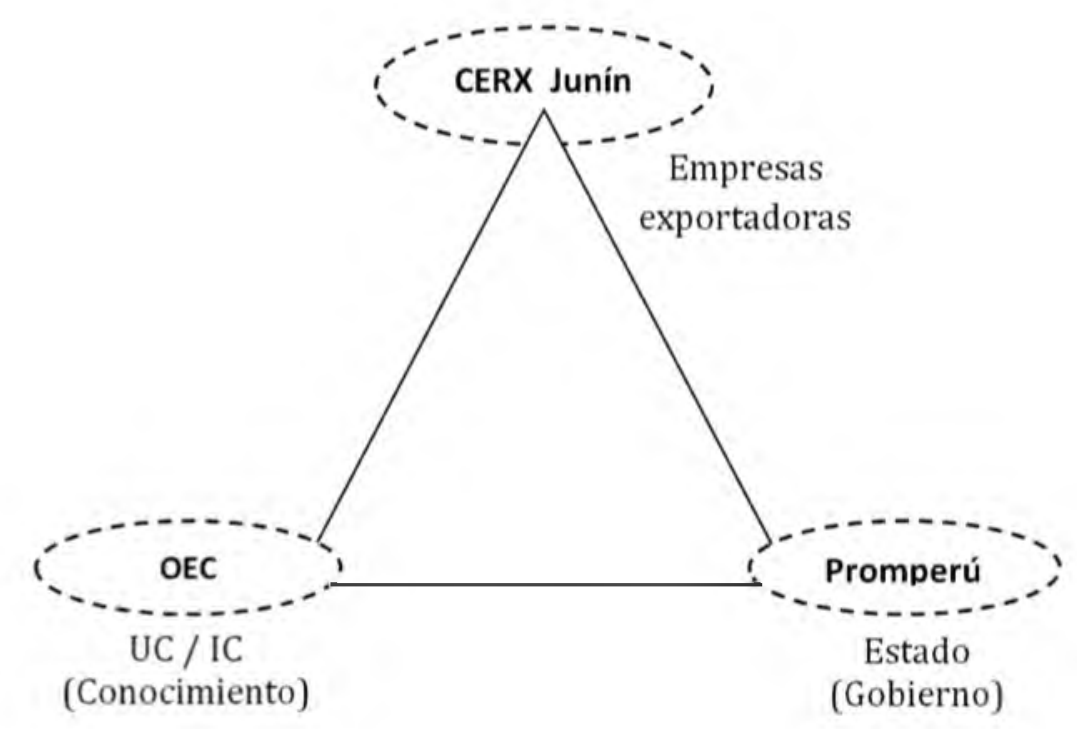

Figura $N^{\circ}$ 1: Triángulo macroeconómico del PTGE.

Exportación del Sector Textil-Confecciones dentro del Plan Maestro de Facilitación del Comercio Exterior. En este POS se reconoce a la región Junín con un gran potencial exportador en el sector de confecciones de fibras de pelo fino o alpaca.

En la región Junín, el sector textil en el rubro de prendas de pelo fino o alpaca no ha tenido apoyo significativo por parte de instituciones del Estado, sin embargo ello no ha anulado el enorme potencial de los productores de este sector; todo lo contrario, es un gremio empresarial que se ha ido consolidando por iniciativa e impulso propio, a pesar de la problemática propia de las microempresas en el Perú (poco acceso a la capacitación, asistencia técnica, etc.). Esto por la escasez de recursos y la priorización que ellos realizan; no obstante, vieron en el PTGE una gran oportunidad para acceder a asistencia técnica, que fue brindada por los alumnos de los últimos semestres y los docentes de diferentes carreras profesionales tanto de la Universidad Continental como del Instituto Continental.

Antecedentes de programas tripartitos (Estado, empresa privada, instituciones educativas) como el que ha sido desarrollado en el PTGE a favor del gremio textil del CERX Junín, no han sido encontrados. La labor de
Promperú al ser un ente de promoción de exportaciones, tiene dentro de sus objetivos institucionales, sin embargo, el apoyo vía capacitación a empresas con potencial exportador, y en función de ello ha venido apoyando a este gremio empresarial.

El objetivo general del estudio fue incrementar el nivel de competitividad de empresas del sector textil con potencial exportador. Los objetivos específicos plantean desarrollar las áreas más críticas de gestión empresarial de acuerdo con el resultado del mapa de competitividad, a través del apoyo de los diferentes programas de Promperú; asimismo aumentar las ventas de exportación a través del uso de herramientas tecnológicas, el posicionamiento en el mercado nacional e internacional.

\section{MATERIAL Y MÉTODOS}

El diagnóstico competitivo (5) inicial realizado dentro del PTGE a las empresas que participaron en este se muestra en la tabla 1.

De acuerdo con el diagnóstico inicial de competitividad desarrollado por Promperú (6), se observa que las áreas más débiles 
son los sistemas de información, con una calificación de 2,09; gestión ambiental, con 2,34; y comercialización, con 2,82. Las áreas con mayores fortalezas son contabilidad y finanzas, con 3,29 de calificación; recursos humanos, con 3,29; y aseguramiento de la calidad con 3,18.

El promedio de las empresas del PTGE fue de 2,96 (sobre un total de 5 puntos), que representa el $59 \%$ de competitividad con la que empezaron las empresas de dicho programa. Este desempeño se muestra en la figura 2.

El PTGE se inicia cuando Promperú (6) realiza un diagnóstico de competitividad y un plan de mejora a 15 empresas socias del CERX Junín (tabla 2).

La principal dificultad para poner en marcha la mejora continua de las empresas del PTGE fue la implementación de los diferentes programas por la falta de conocimientos por parte de los propietarios de las empresas. Frente a ello, se decidió insertar capital humano de bajo costo (estudiantes universitarios y técnicos) que permitan desarrollar las áreas más críticas de la empresa en el corto plazo. A ello se sumó como asesores a docentes de la Universidad Continental y del Instituto Continental, con el fin de darle el soporte
Tabla $N^{\circ}$ 1: Tamaño de la población y muestra del estudio

\begin{tabular}{lcc}
\hline & \multicolumn{2}{c}{ Calificación de } \\
\hline & \multicolumn{2}{c}{ las empresas } \\
\hline 1. Planeamiento estratégico & 2,99 & $60 \%$ \\
2. Producción y operaciones & 3,09 & $62 \%$ \\
3. Aseguramiento de la calidad & 3,18 & $64 \%$ \\
4. Comercialización & 2,82 & $56 \%$ \\
5. Contabilidad y finanzas & 3,29 & $66 \%$ \\
6. Recursos humanos & 3,29 & $66 \%$ \\
7. Gestión ambiental & 2,34 & $47 \%$ \\
8. Sistemas de información & 2,09 & $42 \%$ \\
\hline Promedio de las empresas & 2,96 & $59 \%$ \\
\hline
\end{tabular}

académico a las decisiones que se tomarían en coordinación con los propietarios de las empresas.

Para la implementación del PTGE se determinó que el capital humano a insertarse en las empresas esté de acuerdo con el organigrama (figura 3) implementado en cada empresa.

Como se observa, la gerencia estuvo a cargo del propietario de la empresa y a partir de ello se determinó la necesidad de contar con las siguientes áreas: en el área de planificación se asignó a un estudiante de la Escuela de Economía de la Universidad Continental; en el área de producción uno

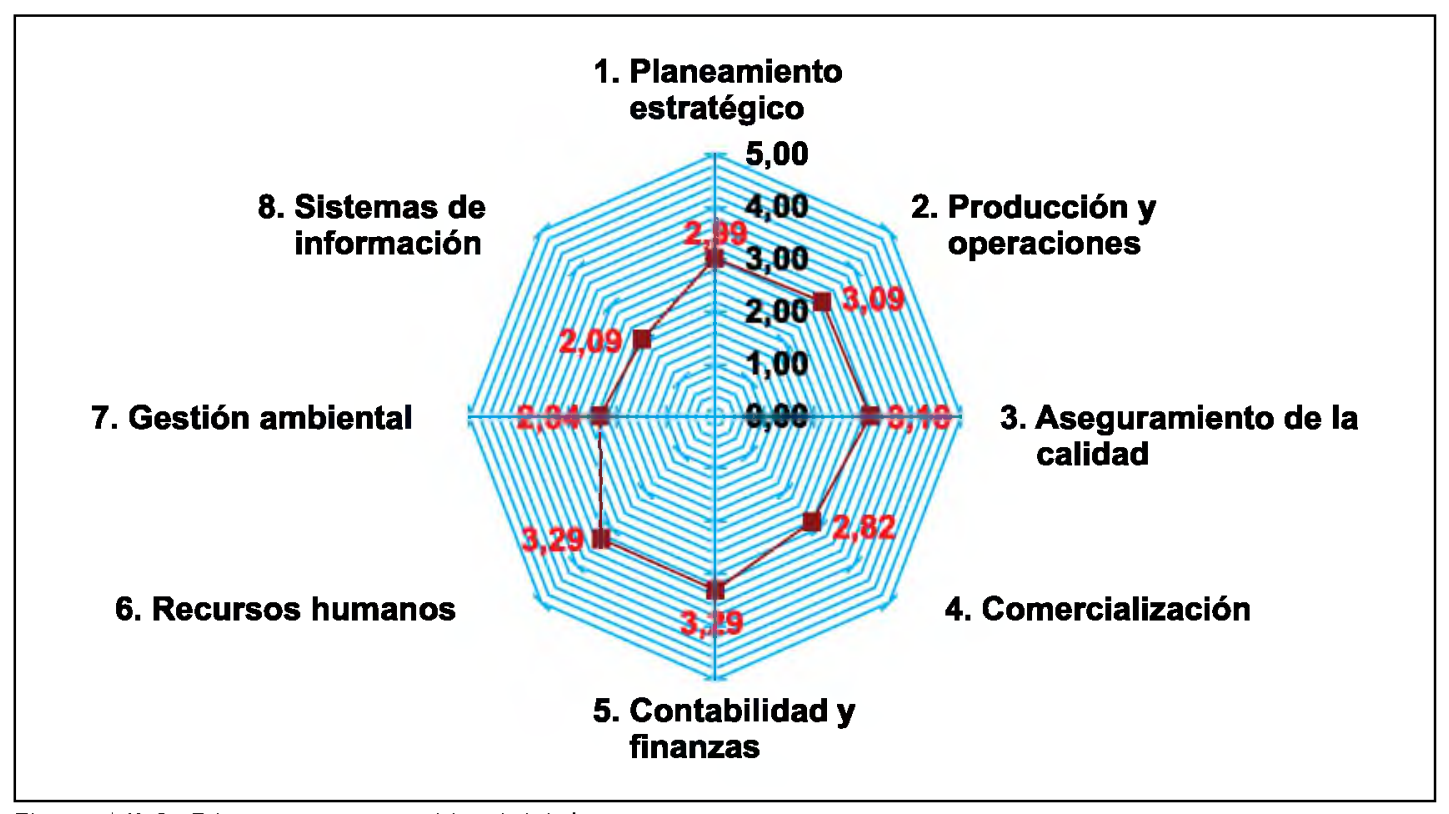

Figura $\mathrm{N}^{\circ}$ 2: Diamante competitivo inicial. 
de la Escuela de Ingeniería de Sistemas; para la supervisión de la producción uno de la Escuela de Ingeniería Industrial; y para el diseño de los productos en la empresa, a una estudiante de la carrera de Diseño de

Tabla № 2: Relación de empresas del PTGE.

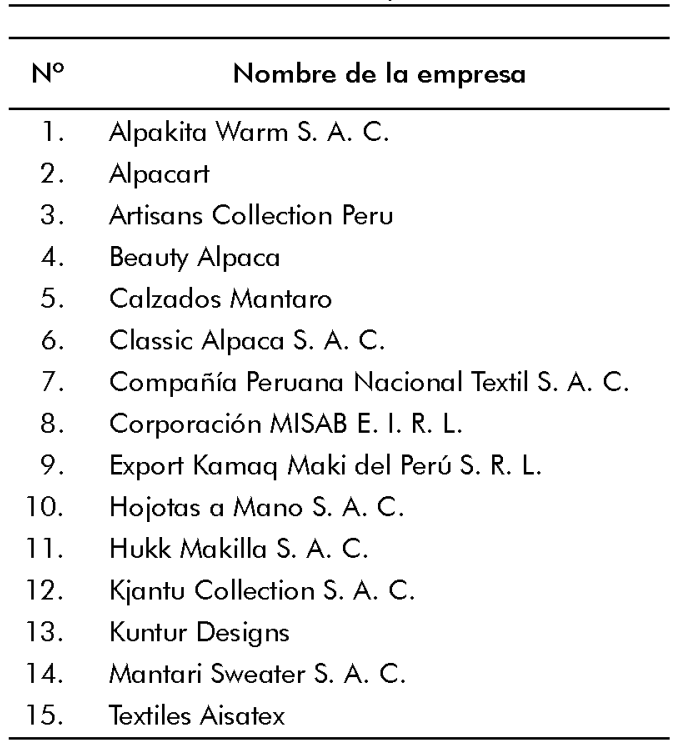

Modas del Instituto Continental. En el área de marketing y ventas fue asignado un estudiante de la Escuela de Administración de la Universidad Continental para apoyar en la comercialización de los productos de la empresa; mientras que en el área de finanzas y contabilidad, un estudiante de la Escuela de Contabilidad para apoyar con el tema contable y tributario en la empresa. En cada caso se asignó a un docente por cada área definida, quien cumplió con la función de asesorar y orientar a los estudiantes. Para la implementación del PTGE se realizó la negociación de responsabilidades de las instituciones conformantes del triángulo macroeconómico: la Organización Educativa Continental, Promperú y el CERX Junín.

Entre las diferentes actividades se realizó la convocatoria a las empresas socias del CERX Junín, a estudiantes de la Universidad Continental y del Instituto Continental de acuerdo con el organigrama elaborado para las empresas del PTGE; también la capacitación de los alumnos seleccionados para realizar prácticas en las empresas del PTGE; la evaluación inicial de las empresas del PTGE; el desarrollo del PTGE ( 9 meses); y los resultados e informe final (figuras $4 \mathrm{y}$ 5).

El desarrollo del PTGE tuvo actividades realizadas por los estudiantes participantes, las que resumimos por trimestres.

En el primer trimestre apoyaron a las empresas en preparar el material necesario para participar en Sur Exporta 2013 en la implementación de los programas 55 y Buenas Prácticas de Comercio Justo,

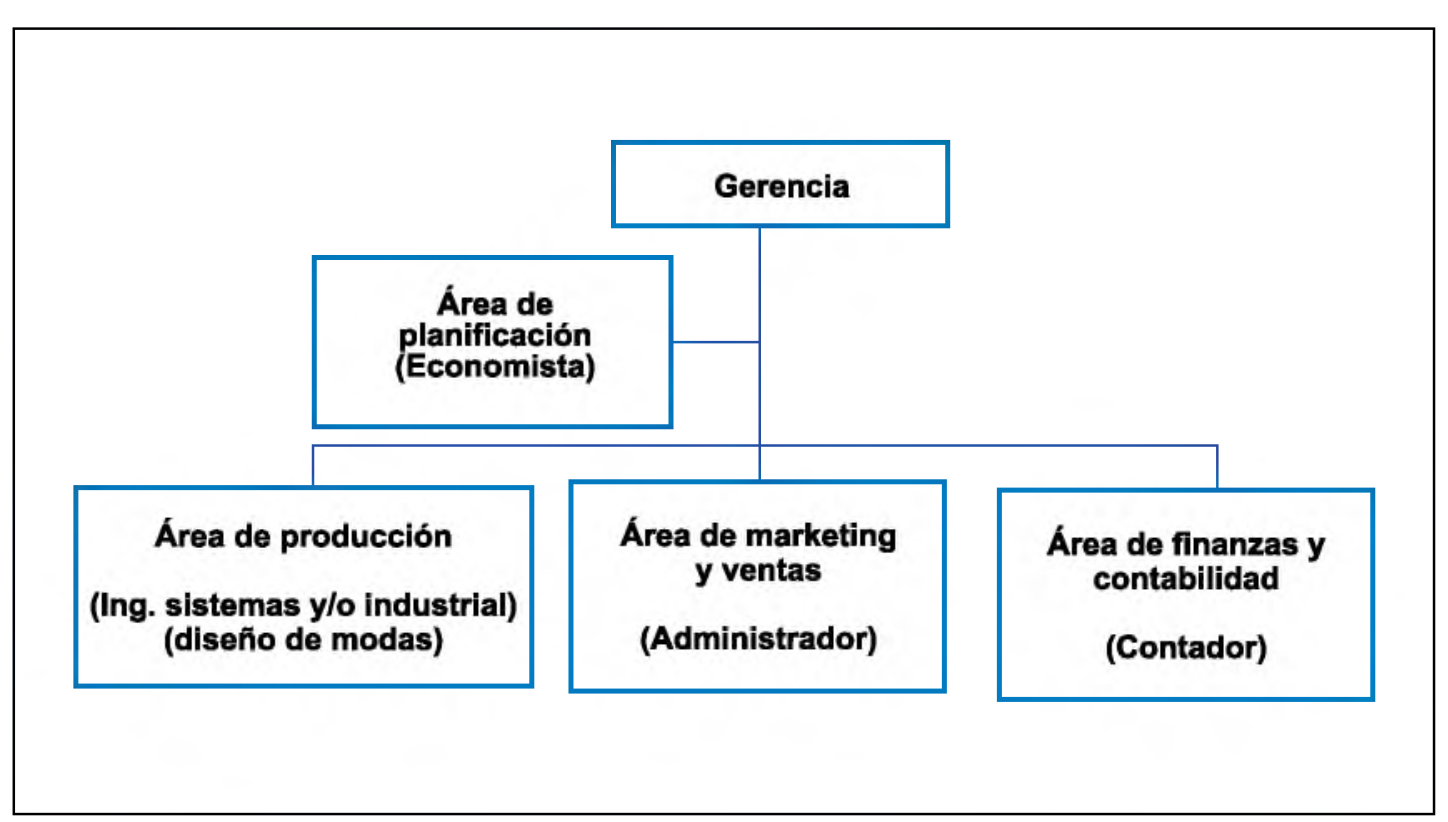

Figura $\mathrm{N}^{\circ}$ 3: Organigrama de las empresas del PTGE. 


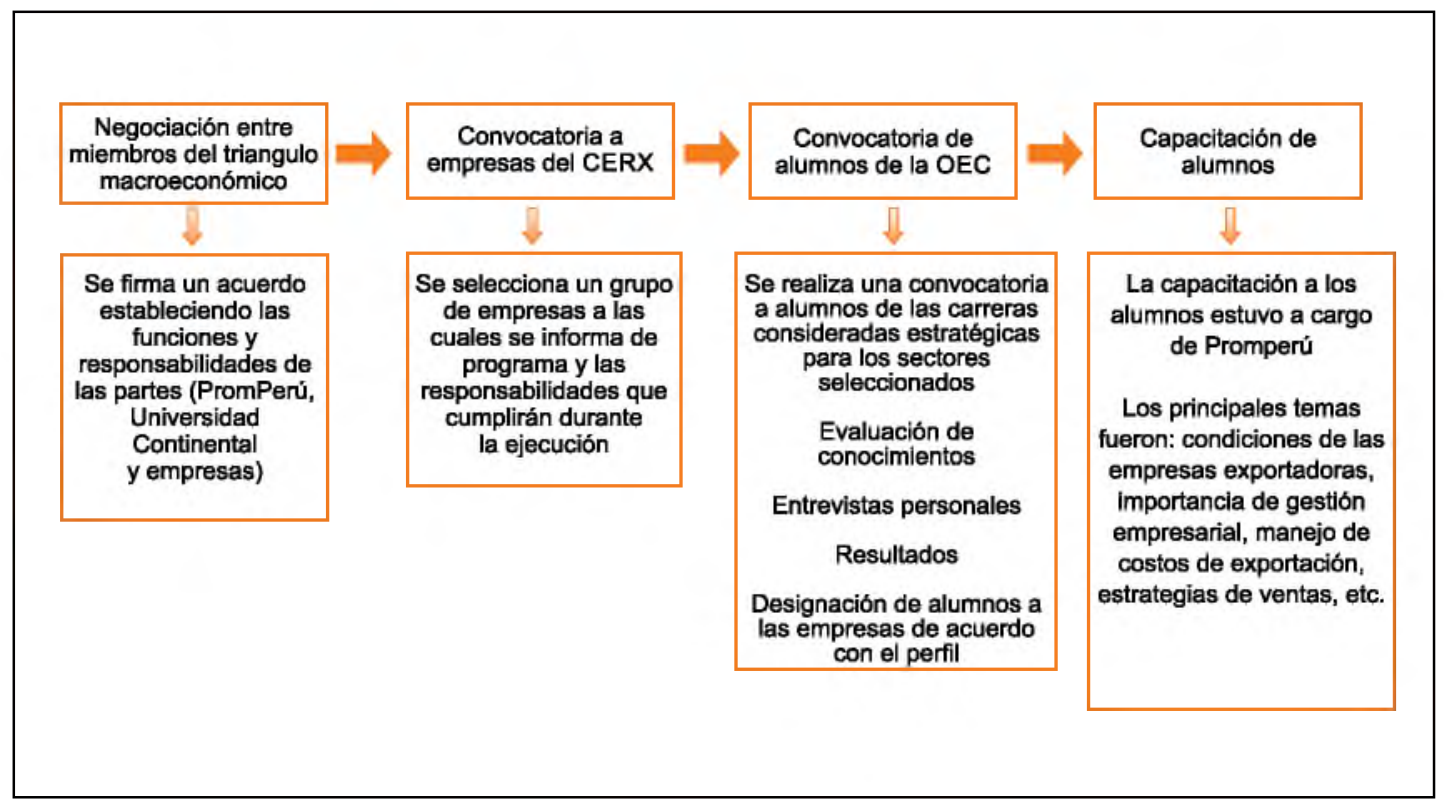

Figura $N^{\circ}$ 4: Desarrollo del PTGE, de la negociación a la capacitación estudiantil.

así como en el desarrollo de tareas del empresario en el programa E-commerce.

En el segundo trimestre fue desarrollado la exposición "Moda Fina" en el Hotel de Turistas de Huancayo; continuó el apoyo en la implementación de los programas $5 S$ y Buenas Prácticas de Comercio Justo, así como en el desarrollo de tareas del empresario en el programa E-commerce para los envíos por Exporta Fácil a las empresas encargadas de preparar el material necesario para participar en la feria Hecho a Mano para Hoteles.

En el tercer trimestre se dio el desarrollo de los puntos críticos del Mapa de Competitividad que no fueron cubiertos en Comercio Justo y 5S; la realización de dos exposiciones "Moda Fina" en la Cámara de Comercio de Huancayo y el auditorio Nuestra Señora del Valle; el apoyo en la implementación del programa Buenas Prácticas de Comercio Justo; y del mismo modo el apoyo para

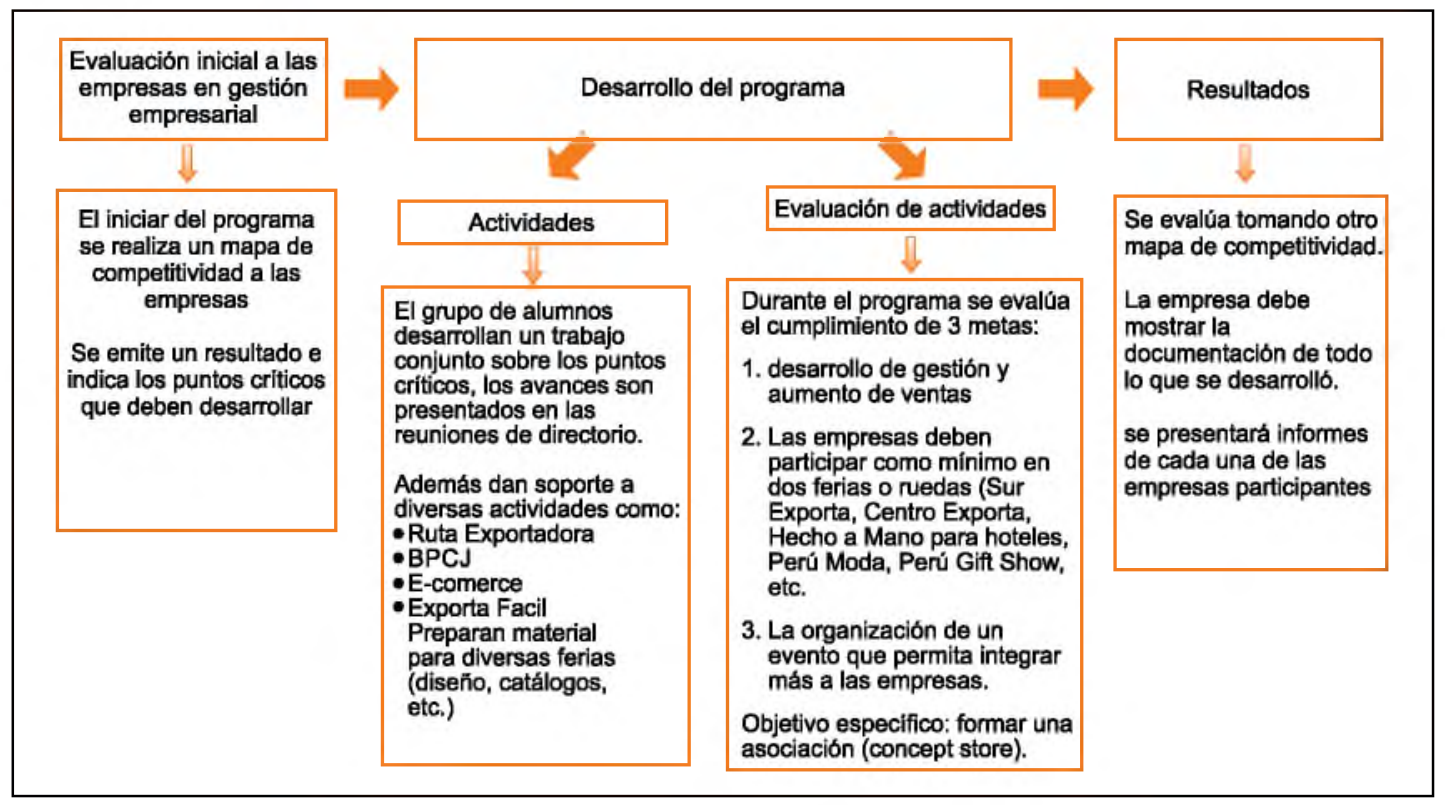

Figura $N^{\circ}$ 5: Desarrollo del PTGE, de la evaluación inicial a la evaluación final. 
los envíos por Exporta Fácil a las empresas encargadas de preparar el material necesario para participar en Perú Moda y Centro Exporta 2014.

Los alumnos también participaron en diferentes actividades nacionales $y$ programas que incidieron en la mejora de la competitividad de las empresas del PTGE. Estas actividades se muestras en la tabla 3.

El consolidado de las diferentes actividades desarrolladas por los estudiantes a favor de las empresas del PTGE se resume en la figura 6 .

La figura 6 muestra la participación de los estudiantes en el PTGE: 11 alumnos fueron contratados en las empresas exportadoras y en proceso de exportación; 4 alumnos viajaron a Sur Exporta 2013 para apoyar al empresario de textiles en la negociación y en el encuentro de negocios "Entre Ojotas y Corbatas", ellos lo hicieron con viáticos pagados por la confianza que generaron a los propietarios de las empresas participantes del PTGE; y solo una alumna apoyó en Hecho a Mano para Hoteles, porque la feria fue durante semana de exámenes finales programados por la universidad.

Estafiguratambién muestraque las empresas
Tabla N ${ }^{\circ}$ 3: Actividades desarrolladas en el PTGE

\begin{tabular}{lc}
\hline $\begin{array}{c}\text { Actividades de } \\
\text { promoción comercial } \\
\text { en las que participaron } \\
\text { las empresas }\end{array}$ & $\begin{array}{c}\text { Programas } \\
\text { desarrollados para } \\
\text { la mejora del sector } \\
\text { exportador }\end{array}$ \\
\hline - Sur Exporta & Ruta Exportadora \\
- Perú Moda & Buenas Prácticas de \\
- Hecho a Mano para & Comercio Justo \\
Hoteles & E-comerce \\
- Expoalimentaría & Exporta Fácil \\
- Centro Exporta & \\
\hline
\end{tabular}

que participaron en Exporta Fácil tuvieron el respaldo de los estudiantes del PTGE. Por ejemplo, la Asociación Llamkasun, para que pueda realizar el envío de muestras de exportación, estableció coordinación entre el alumno, sus asesores y el consultor. Sobre las capacitaciones de E-comerce, el empresario y su alumno del área de administración asistieron a fin de que el universitario pueda dar soporte interno en la empresa y brindar apoyo al gerente en las actividades asignadas por el consultor. Como los alumnos ya formaban parte de la empresa, estos apoyaron en la implantación de los programas $5 S$ y Buenas Prácticas de Comercio Justo; el trabajo motivó una competencia por la implementación, hecho que permitió el logro de mejores resultados en las empresas.

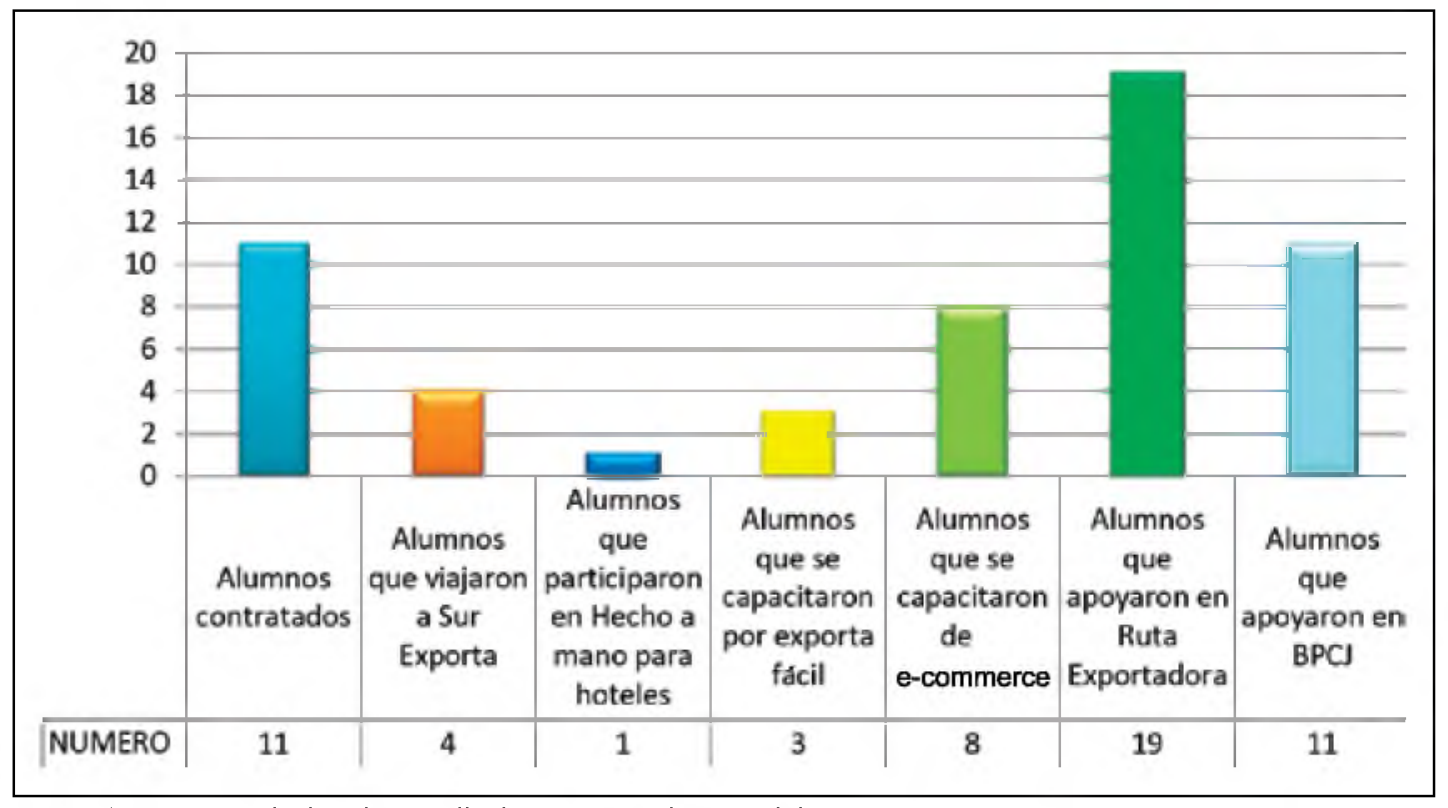

Figura $\mathrm{N}^{\circ}$ 6: Actividades desarrolladas por estudiantes del PTGE. 


\section{RESULTADOS}

Los resultados del PTGE, obtenidos después de 9 meses, muestra que la competitividad de las empresas participantes del programa alcanzó un significativo aumento, entre $73 \%$ y $76 \%$ en todas las áreas, con un porcentaje inferior a $63 \%$ solo en el área de sistemas de información, pero con un promedio general de $73 \%$ (tabla 4), incremento que también se manifiesta en la calificación de crecimiento, como observamos en la figura 7.

Comparativamente, las empresas del gremio textil del PTGE lograron un incremento general de competitividad de $14 \%$, con un aumento de la calificación competitiva de 0,7 décimas, de 2,96 que tuvo en el diagnóstico competitivo inicial a 3,67 alcanzado en el diagnóstico final (tabla 5).

Por áreas, según la tabla 6 y la figura 8 , el incremento de competitividad también fue significativo: un avance de $60 \%$ a $73 \%$ en planeamiento estratégico, de $62 \%$ a $74 \%$ en producción y operaciones, de $64 \%$ a 76 $\%$ en aseguramiento de la calidad, de 56 $\%$ a $74 \%$ en comercialización, de $66 \%$ a $74 \%$ en contabilidad y finanzas, de $66 \%$ a $75 \%$ en recursos humanos, de $47 \%$ a
Tabla $N^{\circ}$ 4: Diagnóstico final de competitividad de las empresas del PTGE.

\begin{tabular}{lcc}
\hline & & \\
\hline & \multicolumn{2}{c}{$\begin{array}{c}\text { Calificación de } \\
\text { las empresas }\end{array}$} \\
\hline 1. Planeamiento estratégico & 3,65 & $73 \%$ \\
2. Producción y operaciones & 3,71 & $74 \%$ \\
3. Aseguramiento de la calidad & 3,80 & $76 \%$ \\
4. Comercialización & 3,70 & $74 \%$ \\
5. Contabilidad y finanzas & 3,69 & $74 \%$ \\
6. Recursos humanos & 3,75 & $75 \%$ \\
7. Gestión ambiental & 3,75 & $75 \%$ \\
8. Sistemas de información & 3,14 & $63 \%$ \\
\hline Promedio de las empresas & 3,67 & $73 \%$ \\
\hline
\end{tabular}

$75 \%$ en gestión ambiental, y de $42 \%$ a $63 \%$ en sistemas de información. Las que más crecieron en competitividad fueron las áreas de gestión ambiental (28\%) y de sistemas de información (21\%). Con ello se cumple uno de los objetivos importantes de esta investigación, que estuvo orientado a contrastar el nivel de competitividad inicial

Tabla $N^{\circ}$ 5: Avance de gestión de la competitividad en las empresas del PTGE.

\begin{tabular}{lccr}
\hline & $\begin{array}{c}\text { Diagnóstico } \\
\text { inicial de } \\
\text { competitividad }\end{array}$ & $\begin{array}{c}\text { Diagnóstico } \\
\text { final de } \\
\text { competitividad }\end{array}$ & Avance \\
\hline Puntaje & 2,96 & 3,67 & 0,7 \\
Porcentaje & $59 \%$ & $73 \%$ & $14 \%$ \\
\hline
\end{tabular}

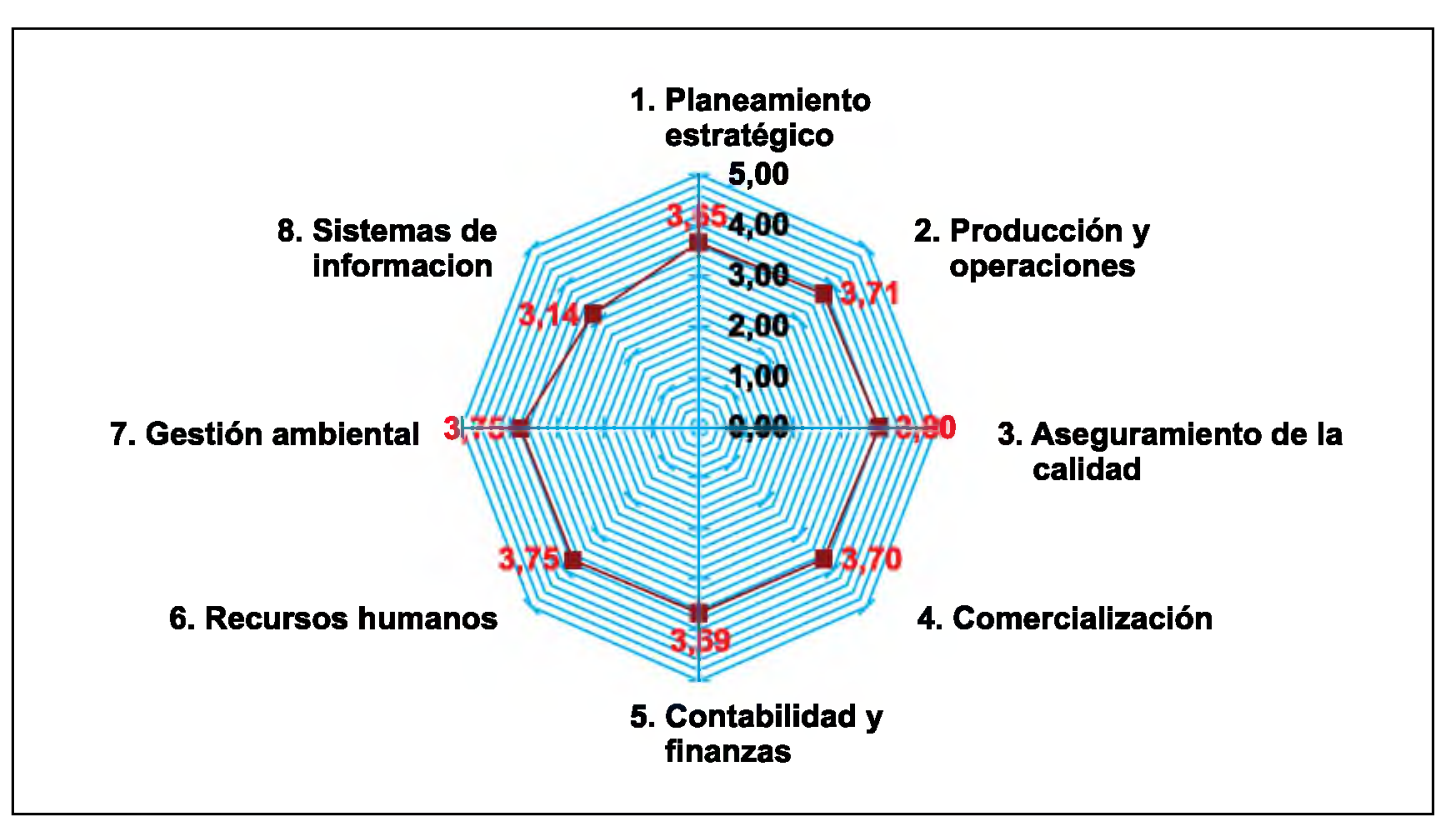

Figura $N^{\circ}$ 7: Diamante competitivo final 
Tabla N ${ }^{\circ}$ 6: Avance de gestión de la competitividad por áreas de las empresas del PTGE.

\begin{tabular}{lcc}
\hline & \multicolumn{2}{c}{$\begin{array}{c}\text { Calificación de } \\
\text { las empresas }\end{array}$} \\
\hline Áreas & Inicial & Final \\
\hline 1. Planeamiento estratégico & $60 \%$ & $73 \%$ \\
2. Producción y operaciones & $62 \%$ & $74 \%$ \\
3. Aseguramiento de la calidad & $64 \%$ & $76 \%$ \\
4. Comercialización & $56 \%$ & $74 \%$ \\
5. Contabilidad y finanzas & $66 \%$ & $74 \%$ \\
6. Recursos humanos & $66 \%$ & $75 \%$ \\
7. Gestión ambiental & $47 \%$ & $75 \%$ \\
8. Sistemas de información & $42 \%$ & $63 \%$ \\
\hline Promedio de las empresas & $59 \%$ & $73 \%$ \\
\hline
\end{tabular}

versus el nivel de competitividad final (7) de las empresas participantes.

\section{DISCUSIÓN}

Se ha demostrado que el PTGE ha logrado los objetivos perseguidos por este programa, toda vez que se llegó a incrementar en $14 \%$ en nivel de competitividad de las empresas que participaron en el PTGE.

El PTGE ha sido el primer programa desarrollado en la Región Junín, que ha permitido sinergias entre el Estado, la empresa y la academia. Con los resultados obtenidos estimamos que deberían desarrollarse más programas como éste que permita interactuar a los alumnos de instituciones educativas superiores en empresas desarrollando actividades pre profesionales que apunte al logro de resultados a todos los participantes.

Por un lado, los empresarios lograron mejorar su nivel de competitividad que les permita acceder al mercado internacional con mayor probabilidad de éxito. Los estudiantes participantes en el PTGE les ha permitido contrastar y poner en práctica sus conocimientos de acuerdo a su especialidad. El Estado a través de Prom Perú ha contribuido a mejorar sus servicios a su público objetivo, en este caso, empresas con potencial expotador.

Mención aparte es la relevancia de este programa para el Instituto Continental y la Universidad Continental, porque como resultado de este programa se ha logrado validar el Plan de Estudios de las especialidades participantes en el PTGE, esto porque se ha comprobado la pertinencia de las áreas de estudio impartido a los estudiantes. Pero también, ha permitido realizar mejoras y reforzamientos en el Plan de Estudio de las Escuelas de Economía en tópicos de Planificación y en la Escuela de Administración en tópicos de Gestión de Ventas. Por otro lado, en la Escuela de Ingeniería de Sistemas e Informática se ha demostrado que las competencias blandas en los alumnos de esta especialidad es un imperativo a desarrollarse.

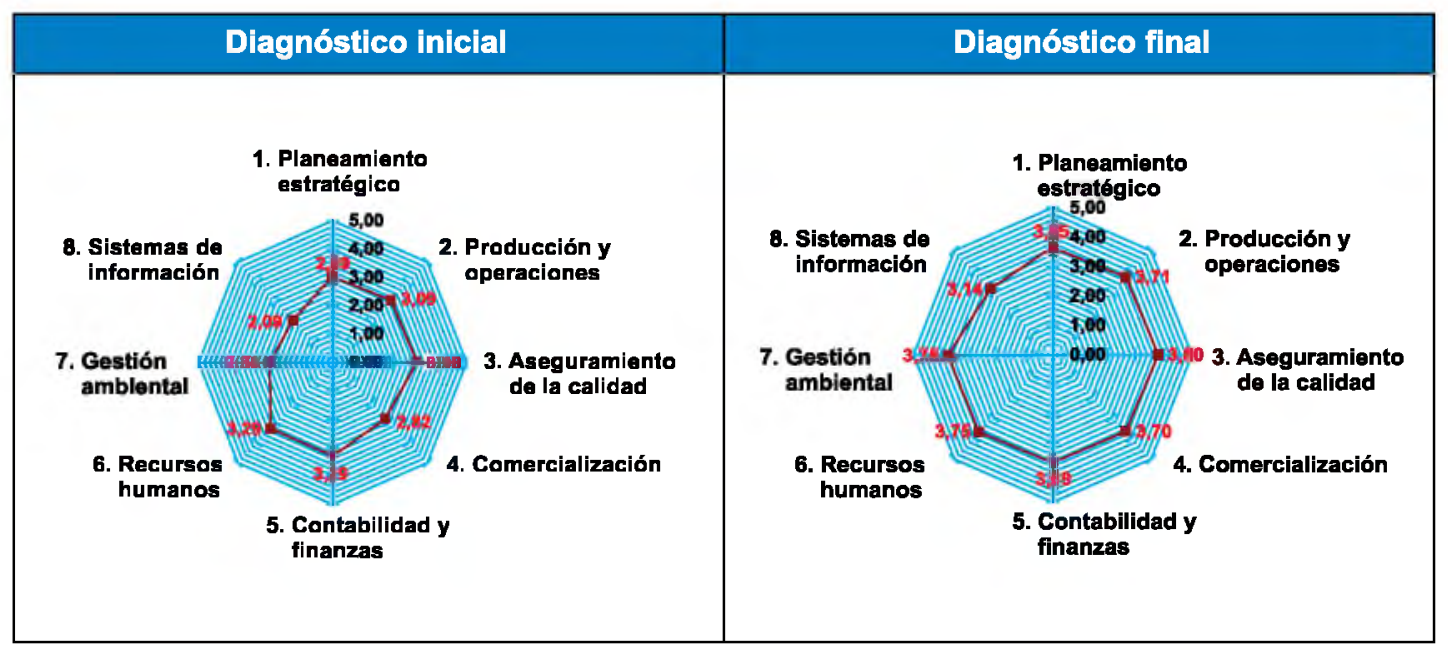

Figura $N^{\circ} 8$ : Resultado comparativo de competitividad por áreas de las empresas del PTGE. 
Algunas conclusiones a las cuales se han arribado son:

Se incrementó el nivel de competitividad de empresas participantes del PTGE en un $14 \%$.

Se incrementó porcentualmente la competitividad de las empresas del PTGE: $18 \%$ en Comercialización, $21 \%$ en Sistemas de Información y $28 \%$ en Gestión Ambiental. Por citar las áreas donde se alcanzó un mejor desempeño.

Se incrementó las competencias laborales de especialidad y competencias blandas en los estudiantes participantes en el PTGE. Se mejoró las mallas curriculares de las Escuelas de Economía y Administración como resultado las labores pre profesionales realizadas por los estudiantes.

\section{Agradecimientos}

Al Ing. Justo Huamán, presidente del Consejo Ejecutivo Regional Exportador Junín (CERX Junín) por la predisposición para convencer a los empresarios para la participación de éstos en el PTGE; y al Econ. Aldo Palomino, coordinador macrorregional de Promperú, por la iniciativa y supervisión de este programa.

\section{REFERENCIAS BIBLIOGRÁFICAS}

1. Abdel G, Romo D. Sobre el concepto de competitividad. Serie de documentos de trabajo en estudios de competitividad. México: ITAM; 2004.

2. García E. Competitividad en el Perú: Diagnóstico, sectores a priorizar y lineamientos a seguir para el período 2011-2016. GCG. 2011; 5(1): 112141.

3. Indacochea A, Ascencio O, Carranza F, De los Ríos L, Wendorff J. Junín competitivo: Valle del Mantaro. 1 ${ }^{\mathrm{a}}$ ed. Lima: Doe Run Perú; 2005.

4. Ministerio de comercio exterior y turismo. Plan estratégico regional exportador, Región Junín. Huancayo:
MINCETUR; 2005.

5. Padilla R. Instrumento de medición de la competitividad. México: Cepal; 2006.

6. Test de competitividad [Internet]. Lima: Sistema Integrado de Información de Comercio Exterior; 2012 [Citado 20 de febrero de 2014 ]. Disponible en: http:// media.peru.info/siicex/resources/ rutaexportadora/08-RE-TEST-DECOMPETITIVIDAD.pdf

7. Dussel E. Un análisis de la competitividad de las exportaciones de prendas de vestir de Centroamérica utilizando los programas y la metodología CAN y MAGIC. México: Cepal; 2001. 